\title{
An analysis of players and bots behaviors in MMORPG
}

\author{
Yutaro Mishima \\ The University of Tokyo \\ Tokyo, Japan \\ Email: kohaku@hongo.wide.ad.jp
}

\author{
Kensuke Fukuda \\ National Institute of Informatics \\ Tokyo, Japan \\ Email: kensuke@nii.ac.jp
}

\author{
Hiroshi Esaki \\ The University of Tokyo \\ Tokyo, Japan \\ Email: hiroshi@wide.ad.jp
}

\begin{abstract}
Bot", automatic robot software, has been one of most serious problems in Massively Multiplayer Online Role Playing Game (MMORPG). Bots earn much more money in a virtual world than human players, and finally collapse balance and fairness of the MMORPG. At present, many techniques have been applied to detect and exterminate bots. However, they have a common problem that the technique effective in a certain MMORPG is not equally effective in other MMORPGs, thus no general method to detect bots has been established. Toward establishing such general technique, we analyze behavioral patterns of human players and bots in server-side game log data with two commonly available features characterizing users (human players and bots): location-based information (i.e., speed of players) and action frequency information (i.e., action count per fixed time slot). The main findings of our analysis are as follows: (1) The variation of the speed of bots is smaller than that of humans (i.e, the movement of bots is more efficient). However, the discriminative power of this location-based feature is not so significant in MMORPG, while it showed good performance in First Person Shooting (FPS) game. (2) Action count and battle count indicate more discriminative power than the speed feature. In particular, the action count is more robust than battle count against the size of the time slot.
\end{abstract}

Index Terms-MMORPG, Bot detection, Data mining

\section{INTRODUCTION}

A large number of Massively Multiplayer Online Role Playing Games (MMORPGs) have been more popular all over the world. "World of Warcraft", for example, is one of the most popular MMORPGs with over 12 million active users [1]. However, several serious problems have been pointed out in MMORPG, such as bots, Real Money Trading (RMT) [2], and cheating. Bots are specialized software that play game automatically, continuously, and simultaneously without human. They can earn much more money or valuable items, by battling against monsters repeatedly, than humans. This leads a collapse of fairness in the game and finally the game gets totally unexciting for most human players. For this reason, how to cope with bots is a serious challenge in MMORPG.

Many companies operating MMORPGs cope with bots by patrolling their game field with some scripts for detecting bots. Moreover, many methods are proposed to detect bots automatically with log data or game traffic data [3]-[10]. Nevertheless, there is no general method to exterminate bots because patrolling is too costly tasks and the methods effective to one MMORPG cannot be directly applicable to other
MMORPGs because of the diversity of game system, program, and communications protocol among MMORPGs.

In this paper, toward establishing more general bot detection, we first carefully focus on behavioral patterns of human players and bots with two common features characterizing humans and bots: location-based information and action frequency information. The former is based on the assumption that bots move efficiently than human players in the game field in microscopic level, and the assumption of the latter is that the number of battles by bots per a time interval is optimal and its variation is small in macroscopic level. We apply the two features to game logs collected at one MMORPG in Japan in order to investigate the discriminative power of these features for bot detection. Our results show that some of the commonlyused behavioral patterns appeared in humans and bots are good metrics to distinguish them. The main findings of our analysis are as follows: (1) The variation of the speed of bots is smaller than that of humans (i.e, the movement of bots is more efficient) in terms of the location-based information. However, the discriminative power of this location-based feature is not so significant in MMORPG, while it showed good performance in First Person Shooting (FPS) game. (2) Action count and battle count indicate more discriminative power than the speed feature. The mean of the frequency for bots is larger than that for most human players, and in particular, the variation of the frequency for bots is small. Furthermore, the action count is more robust than battle count against the size of the time slot.

The rest of the paper is organized as follows. In Section 2, we present some research related to the paper and determine what feature values we use. In Section 3, we describe some conditions in analyzing log data. In Section 4, results and findings of our analyze are presented. In Section 5, we discuss the results and findings. And finally, Section 6 concludes the paper and states our future works.

\section{RELATED WORKS}

In this section we present some related work in the characterization of behavior of humans and bots. The recent literature is broadly catergorized into four approaches: action-based, location-based, input-based, and traffic-based one.

Suznjevic et al. [3] focused on actions taken by human players in a MMORPG. They categorized players' actions in a MMORPG into several types and analyzed frequency of each 
action type for each human player. They pointed out that the routine of human players is similar and they prefer to play with other human players rather than play alone. Thawonmas et al. [4] also categorized the actions in another MMORPG and analyzed how many action types each human player and each bot takes. They pointed out that bots tend to take some actions more intensively than human players.

Chen et al. [5] focused on location information of users. They analyzed moving distance of users per unit time in a First Person Shooting(FPS) game field and pointed out that the distribution of the moving distance of bots is different from that of human players in the game field, meaning that the movement of bots is more regular than that of human.

Gianvecchio et al. [6] focused on input pattern of a mouse or a keyboard by users and analyzed various feature values related to the input. Although humans and bots are distinguishable with the input-related feature values successfully, the method requires detailed movement data of users thus the size of $\log$ data could be huge.

Chen et al. [7] analyzed some feature values in traffic data clients transmit to a game server, e.g. packet rate and RTT. Similar to the input-based method, this method is not directly applicable to other MMORPGs because the method needs a huge volumes of raw level packet data.

Clearly, the bot detection methods have a trade-off between the richness of behavioral features and the difficulty of collecting and maintaining log data. In this sense, the first two approaches focus on more commonly available features than the latter two approaches. However, the first two approaches have been independently investigated and have been applied to one or two MMORPGs. In the following sections, we focus on the usefulness and generality of location-based and actionbased approaches.

\section{DATASET AND FEATURES}

In our analysis, we used a set of game log data recorded at a game server of a popular 3D action MMORPG consisting of over 100000 active players in Japan ${ }^{1}$.

\section{A. Log format}

The server log file is composed of tuples $(<$ timestamp $>$, $<$ action-type $>, \quad<$ player's information $>, \quad<$ location information $>$ ) triggered by a player's action.

- Action-type: The actions, e.g. login, logout, battle with others, trading items, changing location, are classified into 310 categories.

- Time stamp: the current time in the real world is recorded in millisecond-order for each action.

- Player's information: Nickname, player ID, account ID, level, class, race (species).

- Location information: Location on the 3D coordinates in the game field, ID of map and server ID in which the player plays.

\footnotetext{
${ }^{1}$ We cannot disclose the title of the MMORPG due to the contract with the company operating this MMORPG.
}

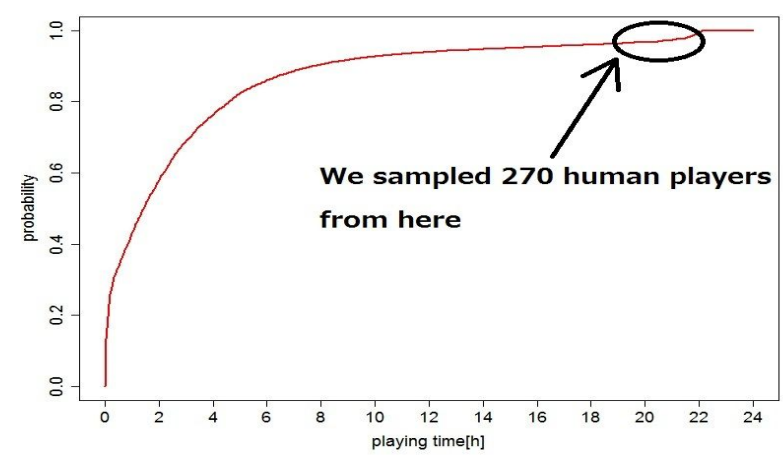

Fig. 1. CDF of players' playing time in a server

\section{B. Dataset}

We analyze a 24-hours log data recorded on Jan. 2012 at one of the game servers. This log data includes both human players and bot data. Administrators of the MMORPG manually detected and actually banned 13 players as bots in the log data. The average playing time of these bots was 23.4 hours, thus, they worked without rest in the day. Fig.1 shows the cumulative distribution function (CDF) of player's playing time in the game server. $\mathrm{X}$-axis represents playing time in the day and $y$-axis represents its cumulative distribution. This figure illustrates that a small number of players play for a long time, while the median playing time after removing nonactive users was about 2 hours. Considering the playing time of bots, we sampled top 270 players in playing time as human players. Therefore, we analyze 270 human player's log data and 13 bot's log data.

\section{Feature selection and Methods}

Here, we explain the feature extraction from the log data and the analysis method in order to distinguish behavior of bots from that of all human players.

1) Location-based information: We first focus on a location-related feature value on the $3 \mathrm{D}$ coordinates in the game field. Moving distance per unit time is known to be good features for bot detection in FPS game [5]. The assumption based on this feature is that the movement of bots is optimal but that of humans has more variation due to randomness of physical actions of humans. We normalize this feature as "speed", as follows:

$$
\text { Speed }=\frac{\text { distance }}{\text { time }},
$$

where the distance is the one between two consecutive actions of a player and the time is the difference of the two time stamps in the log. The bot is programmed optimally, so we expect that the variation of the speed feature in bot is smaller than that in human.

For analysis, we first draw histograms of the speed feature for each human and bot, then observe the difference between them. After we compare the mean and the variance of the 
speed for humans and those for bots, we calculate the coefficient of variation (CV) of the speed for each human and bot. CV is a normalization of the variance, and is used for comparing variation of samples with different means. The definition of $\mathrm{CV}$ is given as:

$$
C V=\frac{\text { standard deviation }}{\text { mean }} \text {. }
$$

A smaller $\mathrm{CV}$ corresponds to a smaller variation of a given dataset, vice versa. As the final step, we evaluate the discriminative power of the speed feature with several performance indices. We draw a commonly used Receiver Operating Characteristic (ROC) curve for varying a threshold value of $\mathrm{CV}$, which is a plot of False Positive Rate (FPR) and True Positive Rate (TPR). ROC curve is an indicator for measuring performance of classification algorithm. In this analyze, we define bot as positive and human as negative because our goal is to detect bots. One performance index with ROC curve is the minimum Euclid distance from the coordinate corresponding to the best performance in ROC curve: FPR $=0$ and $\mathrm{TPR}=1$. A smaller distance indicates better performance of classification. Finally, we calculate some other indices, Accuracy, Precision, Recall and F-measure, for evaluation of the discriminative power of this feature value:

$$
\begin{array}{r}
\text { Accuracy }=\frac{T P+T N}{T P+F P+F N+T N}, \\
\text { Precision }=\frac{T P}{T P+F P}, \\
\text { Recall }=\frac{T P}{T P+F N}, \\
F-\text { measure }=\frac{2 * \text { Recall } * \text { Precision }}{\text { Recall }+ \text { Precision }},
\end{array}
$$

where TP, TN, FP and FN are True Positive count, True Negative count, False Positive count and False Negative count, respectively. These indices vary in the range $[0,1]$, and a higher value indicates better performance.

2) Action frequency information: Another feature we focus on is the number of actions per fixed time slot. The background of this feature is based on two related works [3], [4] focusing on player's action and the assumption that bots play in the game world optimally (i.e., they battle against monsters without rest) while human players cannot work optimally for a long time. Thus, the variation of the number of battle actions in bots is expected to smaller than that in humans. Furthermore, the variation of the number of all actions in bots is expected to smaller than that in humans, considering the fact that most of actions by bots are battle. Therefore, we calculate the number of all actions per time slot as "action count", and the number of battle actions per time slot as "battle count" for every human and bot.

We draw histograms of action count and battle count for each human and bot, then check whether there is difference between them. Next, we calculate the mean and the variance of action count and battle count for each human and bot, as we do in Section IV-A. After analyzing the difference between mean and variance of bots and those of humans, we investigate $\mathrm{CV}$ of speed for each human and bot. As the final step, we evaluate the discriminative power of action count and battle count by the performance indices.

\section{RESULTS}

\section{A. Location-based information}

We first discuss the discriminative power of a location-based feature (i.e., "speed") for bot detection. Fig.2 demonstrates an example of the estimated probability density function (PDF) of speed of two players: human (red) and bot (blue). The $\mathrm{x}$-axis represents the speed between two consecutive actions and the $y$-axis represents its estimated density by a kernel density estimation (KDE) with a standard Gaussian kernel. We visually confirm that the shape of the two plots is stretched and resembles each other, suggesting that the discrimination power of this feature is expected to be low.

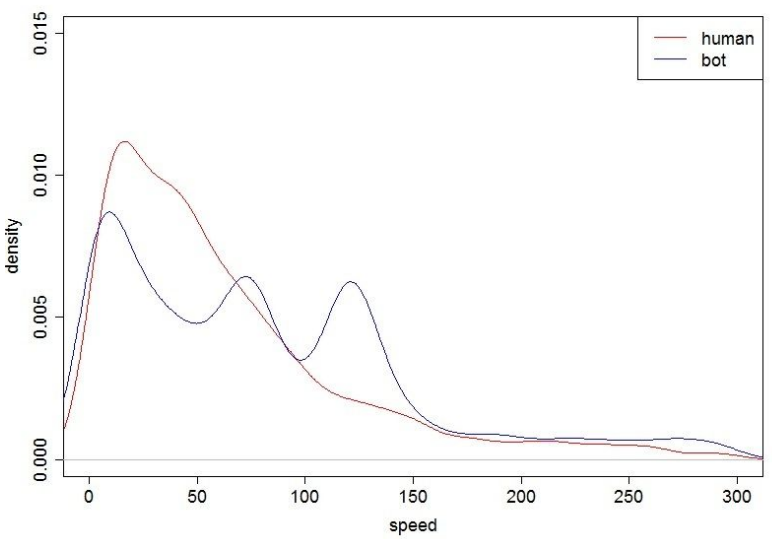

Fig. 2. An example of estimated PDFs of speed feature for two players (a human player and a bot)

Next, Fig. 3 displays a scatter plot of the mean (x-axis) and the variance (y-axis) of speed for each player; Red and blue dots represent humans and bots, respectively. The mean of the human players are widely spread while that of the bots is relatively small. Moreover, the bots are distributed in the lower variance area while many human players are also overlapped in the same area. Thus, this result suggests that the movement of the bots has less variation.

To confirm this findings, we calculated the CV of the speed feature for each human and bot. A smaller CV indicates a smaller variation of a given dataset, as explained above. Fig.4 shows PDFs of CV of the speed feature for all humans (red) and all bots (blue). We could distinguish two curves; a sharp peak for bots and a broader peak for humans. However, two plots are highly overlapped, expecting a poor discriminative power of bots.

Finally, we investigate the discriminative power of the $\mathrm{CV}$ of the speed feature with some indices: The best $\mathrm{f}$-measure value was 0.222 for different thresholds of the $\mathrm{CV}$, and other indices for the best f-measure are listed in Table II. Also, a ROC curve 


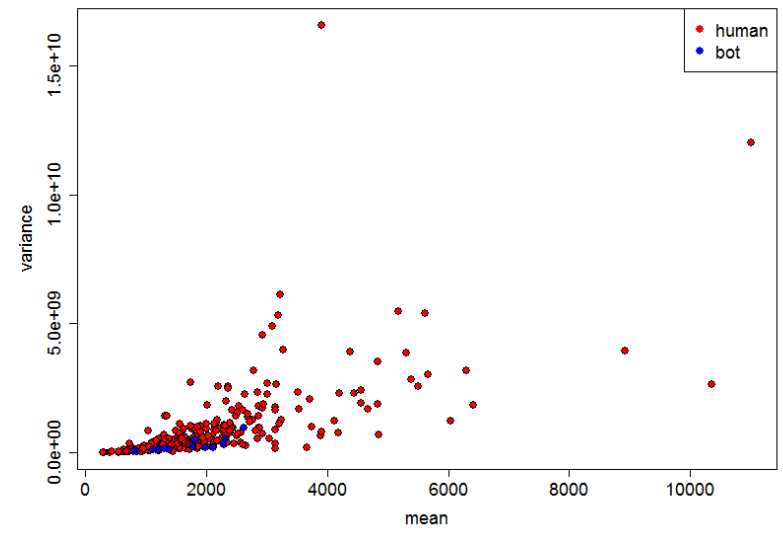

Fig. 3. Scatter plot of speed feature: mean and variance

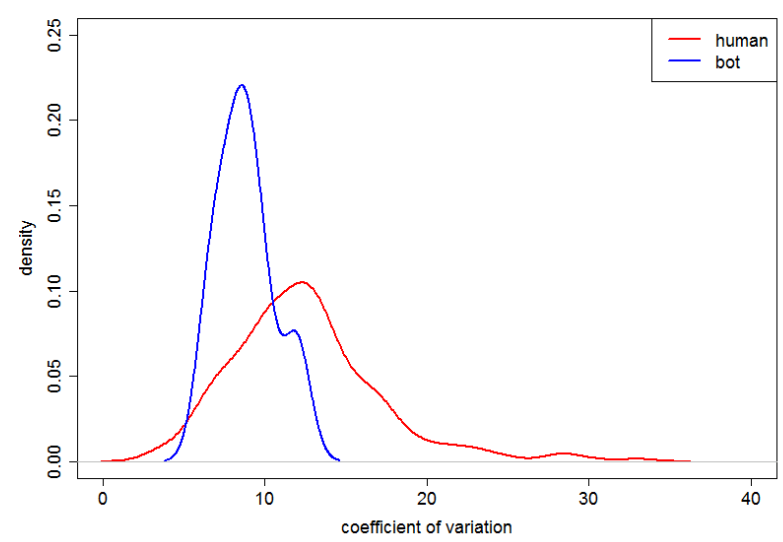

Fig. 4. Estimated PDF of $\mathrm{CV}$ of speed feature

of the $\mathrm{CV}$ of the speed feature is presented in Fig.5. The $\mathrm{x}$ axis and the y-axis represent FPR and TPR, respectively. The minimum Euclid distance from the best coordinate $(0,1)$ was 0.327, and FPR and TPR for this point are 0.289 and 0.846, respectively.

The results demonstrated that the speed feature indicates low discriminative power to distinguish bots from human players in this MMORPG. A plausible reason of this low discriminative power is that this speed feature is originally developed for a FPS game, not for MMORPG. We conclude that humans and bots in MMORPG are less different in terms of speed than those in FPS game.

\section{B. Action frequency information}

TABLE I

BREAKDOWN OF ACTION TYPES FOR HUMAN PLAYERS AND BOTS

\begin{tabular}{|c|c|c|c|c|}
\hline Action type & Battle & Item & Interact & Other \\
\hline Humans & $52.5 \%$ & $25.7 \%$ & $1.27 \%$ & $20.5 \%$ \\
\hline Bots & $69.0 \%$ & $17.2 \%$ & $0.14 \%$ & $13.7 \%$ \\
\hline
\end{tabular}

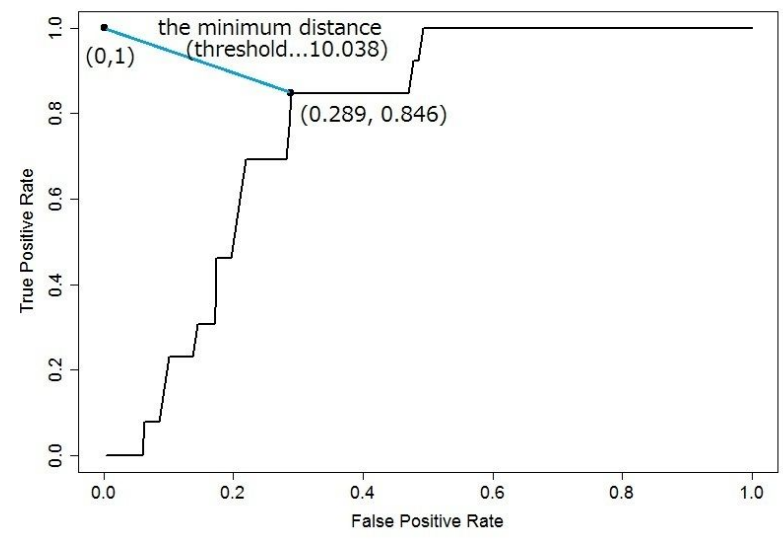

Fig. 5. ROC curve of $\mathrm{CV}$ of speed feature

We first examine the breakdown of the action types for human players and bots. Table I lists the frequency of each action type, battle, using item, interact with other players (e.g. trading, composing a group) and other actions, for human players and bots. As expected, the ratio of battle for bots is $30 \%$ higher than that for humans, meaning that bots concentrates on battle to earn more money while human players take $50 \%$ of non-battle actions. Our preliminary analysis, however, showed that the ratio of battle and that of item were not significant features for bot detection due to large overlaps.

Next, we investigate the discriminative power of the frequency of actions and battles. We first calculate the distribution of action count and battle count per a fixed time slot (e.g. $300[\mathrm{sec}]$ ), for every human player and bot. Fig.6 demonstrates an example of the estimated PDF of action count and battle count per $300[\mathrm{sec}]$. The $\mathrm{x}$-axis represents action (or battle) count and $y$-axis represents its estimated density. Red and blue plots represent PDFs of human and that of bot, respectively, and solid and dashed lines show the difference between the action count and battle count. We find that mean of action count and battle count of bots are greater than those of humans. This means that the bots worked efficiently as expected though they still have some non-active time near 0 . In contrast, the shape of action count and battle count of bots is narrower than that of humans. This fact indicates that bots always take actions more frequently and optimally than humans because humans get tired, chat with others and get away from keyboard for some tasks.

We also find the difference of the mean and the variance of action count and battle count between bots and humans in Fig.7 and Fig.8. In the both scatter plots, the $\mathrm{x}$-axis represents the mean of the counts and the $y$-axis represents the variance of the counts for humans (red) and bots (blue). Almost all of bots are distributed at the lower right area where the variance is small and stable even for large means. On the other hand, the distribution of the human players indicate a positive correlation between the mean and the variance. Some human players are distributed on the right side, indicating that they take as many 


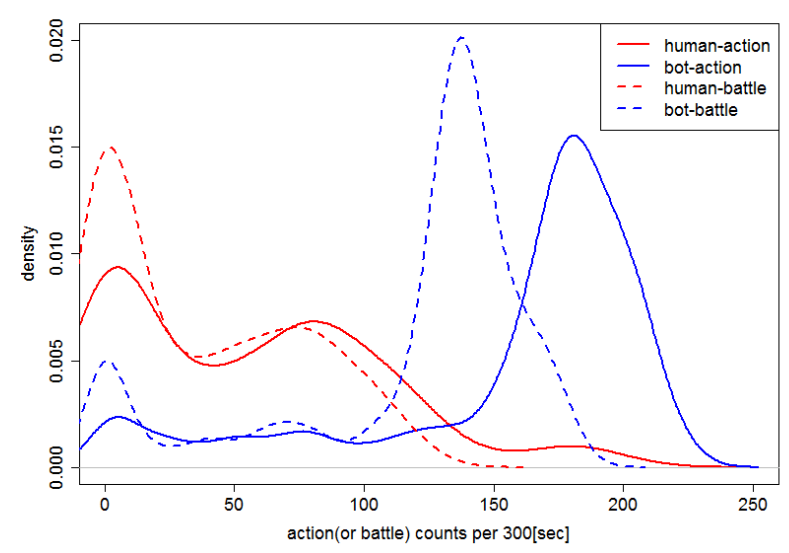

Fig. 6. An example of estimated PDFs of action and battle counts (time slot: $300[\mathrm{sec}])$

actions or battle actions as bots take; They are likely expert human players. However, in most of them, the variance of action count and battle count are larger than those of bots. Therefore, we expect to distinguish bots from humans with the mean and the variance of these two feature values.

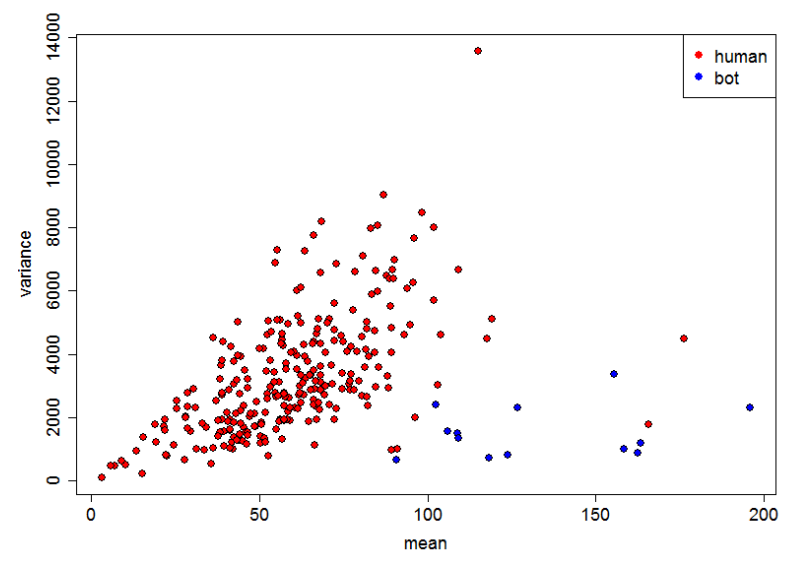

Fig. 7. Scatter plot of action count: mean and variance (time slot: 300[sec])

Next, Fig.9 shows the estimated PDFs of the CV of action count and battle count for humans (red) and bots (blue) per $300[\mathrm{sec}]$. The $\mathrm{x}$-axis represents the value of the CV and $\mathrm{y}$ axis represents its estimated density. Solid and dashed lines represent PDFs of action count and that of battle count. We confirm that bots and humans are differently distributed, thus bots are distinguishable from humans with these two feature values, action count or battle count. Moreover, we can observe that the PDFs of action and battle counts of bots resemble each other because the major action of bots is battle. In contrast, the PDF of action count of humans is more shifted to larger CV than that of battle count showing that the action events except for battle in humans indicate more variation (i.e, randomness).

In order to examine the dependency of the size of the time

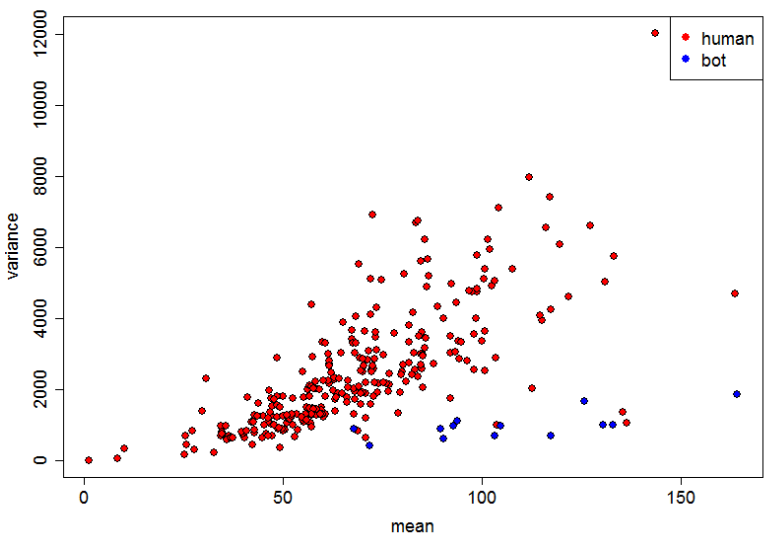

Fig. 8. Scatter plot of battle count: mean and variance (time slot: $300[\mathrm{sec}]$ )

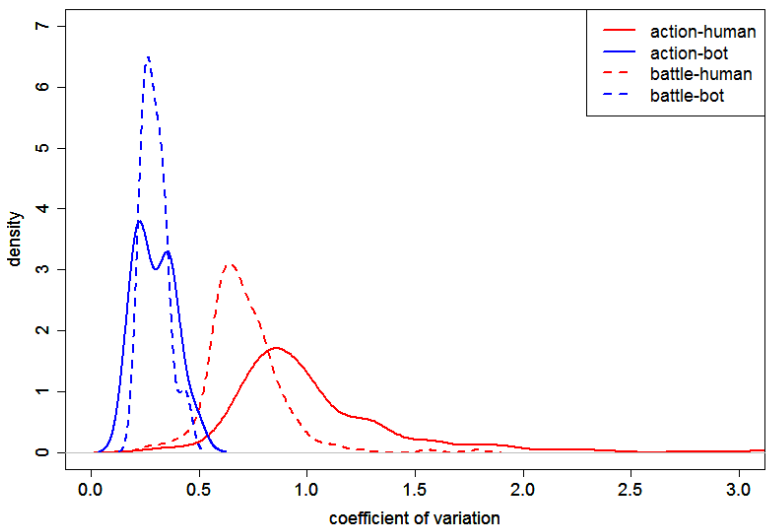

Fig. 9. Estimated PDF of CV of action and battle counts (time slot: 300[sec])

slot on the discriminative power of the features, we analyze CVs of action and battle counts for varying the size of the time slots from $50[\mathrm{sec}]$ to $3600[\mathrm{sec}]$. Fig.10 shows the maximum value of the f-measure for different size of time slot. The $\mathrm{x}$-axis represents the size of the time slot (in log scale) and the y-axis represents the maximum value of $f$-measure for the time slot. Brown and purple lines represent f-measure values of action count and battle count, respectively. The figure demonstrates that the f-measure value of action count is higher than that of battle count in most time slots. Thus, we can detect bots more accurately by analyzing action count than battle count. In action count, the maximum value of the f-measure is 0.867 for time slots from $50[\mathrm{sec}]$ to $210[\mathrm{sec}]$, and $230[\mathrm{sec}]$, while the maximum value of the f-measure is 0.846 at $350[\mathrm{sec}]$ in battle count. This result indicates the robustness of the action count against the size of the time slot. Considering the cost of calculation of CVs, we choose longer time slot (i.e., 230[sec]) for the next analysis.

Finally, we plot a ROC curve for the best time slot $(230[\mathrm{sec}])$ in Fig.11. The plot sharply increases near FPR 


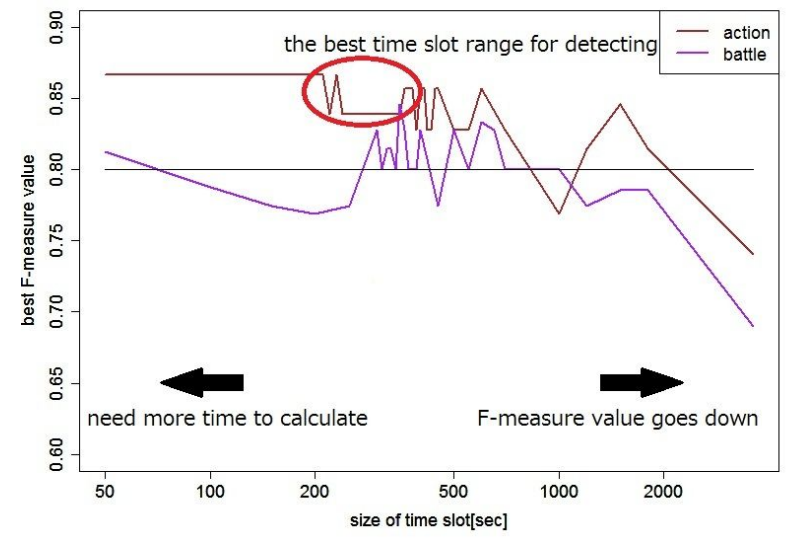

Fig. 10. Variation of f-measure value for different size of time slot

$\approx 0.0$, thus the feature achieves a higher TPR with a lower FPR. The minimum Euclid distance from the coordinate $(0$, $1)$ is 0.0148 which is much smaller than that of speed feature (0.327).

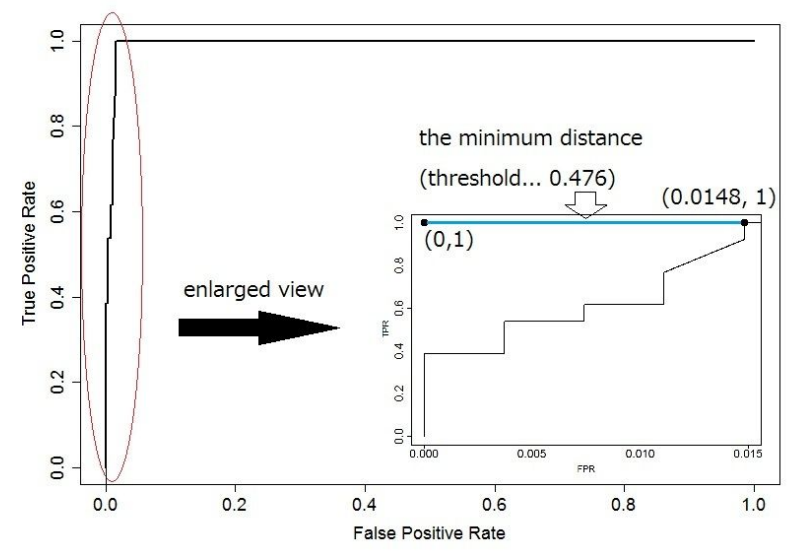

Fig. 11. ROC curve of CV of action count (time slot: 230[sec])

We summarize the results of our performance evaluation for the best $\mathrm{f}$-measure value $(230[\mathrm{sec}])$ in Table II. From this result, we conclude that the action frequency information (i.e., action count and battle count) has better discriminative power to distinguish bots from human players in MMORPG than the location-based information (i.e., speed feature).

TABLE II

SUMMARY OF PERFORMANCE INDICES

\begin{tabular}{|c|c|c|c|}
\hline Indices & Speed & Action count & Battle count \\
\hline Accuracy & 0.777 & 0.986 & 0.986 \\
\hline Precision & 0.132 & 0.765 & 0.846 \\
\hline Recall & 0.692 & 1.000 & 0.846 \\
\hline F-measure & 0.222 & 0.867 & 0.846 \\
\hline
\end{tabular}

\section{DisCusSION}

We analyzed two types of features to distinguish bots from human players. As shown in Table II, the best performance of the action count and battle count is superior to that of the speed feature. The location based feature assumes that the microscopic movement of the bots is optimal while that of the human players has some randomness depending on their physical restriction or the input user interface. On the other hand, the action frequency based features (i.e. action count and battle count) assume that bots are optimal for obtaining money while the concentration of human players to the game does not last so long (e.g., they get tired or they are bored to do battle). In this sense, the action frequency based features are based on more macroscopic and generic behavior of humans and bots, thus we believe that they are also useful in detecting bots in other games.

The size of the time slot and the detectability are a clear tradeoff for real deployment; a shorter time slot requires more processing time but obtains better performance, vice versa. The best performance of the two proposed features is the almost same, however the robustness against the size of the time slot in action count is better than that in battle action as shown in Fig.10. Considering the stability of the size of the time slot, the time slot should be set to less than $250 \mathrm{sec}$ in action count. On the other hand, a smaller time slot works better for faster detection because the method needs a statistically enough number of samples to calculate the mean and variance. Finding the optimal number of samples will be one of our future work.

\section{CONCLUSION}

In this paper, we introduced location-based information (i.e., speed feature) and action frequency information (action count and battle count) of human players and bots to characterize their behaviors in MMORPG. The former is based on the assumption that the microscopic movement of bots has less variation than that of human players, and the assumption of the latter is that the macroscopic action frequency of bots is more stable than that of human players.

We evaluated the discriminative power of these features with a 24-hours log data collected at a major MMORPG in Japan. Regarding the location-based information, we found that human players and bots are not effectively distinguishable by the speed feature in MMORPG. Thus, there are less difference between humans and bots in terms of speed in MMORPGs than those in FPS games. On the other hand, we pointed out that human players and bots are distinguishable in both of action count and battle count, and they are superior to the speed feature.

One of the future work is to apply the proposed features to other MMORPGs in order to verify the discriminative power of the features for bot detection. Another direction of the work will be to develop an online bot detection algorithm based on the proposed features, while we used the offline log taken at the server in the current analysis. 


\section{ACKNOWLEDGMENT}

The authors would like to thank Mangi Leem, Masahito Kikuchi, and Hiroyuki Yanagida for providing us the log data of the MMORPG and helpful discussion. We also thank Koichi Ise for coordinating this collaboration work.

\section{REFERENCES}

[1] GAMASUTRA.COM, "World Of Warcraft Reaches 12 Million Subscribers Worldwide", http://www.gamasutra.com/view/news/ 30845/World_Of_Warcraft_Reaches_12_Million_Subscribers_ Worldwide.php\#.UFPdIciZaJ4.

[2] Hiroshi Itsuki, Asuka Takeuchi, Atsushi Fujita, Hitoshi Matsubara:"Exploiting MMORPG log data toward efficient RMT player detection", ACE'10, pp.118-119(2010)

[3] Mirko Suznjevic, Ognjen Dobrijevic, Maja Matijasevic, "Hack, slash, and chat: a study of players' behavior and communication in MMORPGs?', NetGames '09, p.6, (2009).

[4] Ruck Thawonmas, Yoshitaka Kashifuji, Kuan-Ta Chen, "Detection of MMORPG Bots Based on Behavior Analysis?", ACE '08, pp.91-94, (2008).

[5] Kuan-Ta Chen, Hsing-Kuo Kenneth Pao, Hong-Chung Chang, "Game Bot Identification Based on Manifold Learning", NetGames '08, pp.21-26, (2008).

[6] Steven Gianvecchio, Zhenyu Wu, Mengjun Xie, and Haining Wang, "Battle of Botcraft: Fighting Bots in Online Games with Human Observational Proofs?", CCS '09, pp.256-268 (2009).

[7] Kuan-Ta Chen, Jhih-Wei Jiang, Polly Huang, Hao-Hua Chu, Chin-Laung Lei, Wen-Chin Chen, "Identifying MMORPG bots: a traffic analysis approach", ACE '06, p.9, (2006).

[8] Stefan Mitterhofer, Christopher Kruegel, Engin Kirda, Christian Platzer, "Server-Side Bot Detection in Massively Multiplayer Online Game”, IEEE Security and Privacy, pp.29-36, (2009).

[9] Ibrahim Cevizci, Melike Erol, Sema F. Oktug, "Analysis of multi-player online game traffic based on self-similarity?", NetGames '06, p.9, (2006).

[10] Kuan-Ta Chen, Polly Huang, Chun-Ying Huang, Chin-Laung Lei, "Game traffic analysis: an MMORPG perspective", NOSSDAV '05, pp.19-24, (2005). 